Unfallchirurg 2015 $\cdot 118: 198-198$

DOI 10.1007/s00113-014-2687-z

Online publiziert: 18. März 2015

(c) Springer-Verlag Berlin Heidelberg 2015

\author{
W. Mutschler $\cdot$ T. Geith ${ }^{2}$ \\ ${ }^{1}$ Klinik für Allgemeine, Unfall-, Hand- und Plastische Chirurgie, Klinikum der Ludwig-Maximilians- \\ Universität, Campus Innenstadt, München \\ ${ }^{2}$ Institut für Klinische Radiologie, Klinikum der Ludwig-Maximilians-Universität, \\ Campus Großhadern, München
}

\title{
Das Knochenmarködem in der Unfallchirurgie
}

Sehr geehrte Leserinnen und Leser,

selten hat ein bildmorphologisches Muster wie das im MRT und nur im MRT beobachtbare „Knochenmarködem“ so viel Verwirrung gestiftet. Das fängt schon bei der Sprachbegrifflichkeit an. Knochenmarködemmuster, „bone bruise“, Knochenödem, Knochenkontusion, okkulte Fraktur oder Mikrofraktur sind vielfältig verwendete Wörter zur Beschreibung eines letztlich noch nicht verstandenen Phänomens. Gerade bei Knochenmarködemen nach Trauma liegt rasch der Vorwurf auf dem Tisch, eine Fraktur übersehen und sowohl diagnostisch als auch therapeutisch einen groben Behandlungsfehler begangen zu haben. Mit der zunehmenden Zahl der MRT-Untersuchungen nimmt auch die Zahl der deshalb Beschuldigten bei den Schlichtungsstellen und Gerichten in den letzten Jahren deutlich zu und belastet das Vertrauensverhältnis zwischen Patient und Arzt. Unter anderem deshalb, weil eben im Röntgenbild knöcherne Fissuren (Haarrisse) oft nicht erkannt werden können, okkulte Frakturen mit übersehenen Frakturen gleichgesetzt und Mikrofrakturen zu klassischen Frakturen umdefiniert werden, obwohl eine Kontinuitätsunterbrechung der Makrostruktur des Knochens gerade nicht vorliegt.

Dazu kommt die Diskrepanz zwischen subjektiven Beschwerden, klinischen Befunden und Signalveränderungen im MRT. Zum einen werden Knochenmarködeme als Zufallsbefunde ohne subjektives und klinisches Korrelat diagnostiziert, zum anderen mag das Knochenmarködem Ausdruck einer lang anhaltenden und schmerzhaften Gefügestörung sein, die unmittelbar nach einem Trauma so nicht vorhersehbar war und besonderer Aufmerksamkeit bedarf. Schließlich gibt es auch eine Vielzahl von Knochen- und Gelenkpathologien, bei denen das Knochenmarködem Ausdruck folgenreicher Erkrankungen wie etwa avaskulärer Knochennekrosen und Neoplasien sein kann.

Wie also damit umgehen, diagnostisch und therapeutisch?

Bei unspezifischen Beschwerden und unauffälligen Röntgenbildern zur Differenzialdiagnose (oder auch zur Absicherung) gleich ein MRT anfertigen lassen oder abwarten und symptomatisch therapieren? Und wie weiter vorgehen, wenn ein Knochenmarködem befundet wird?

Im vorliegenden Themenheft wollen wir dazu eine Hilfestellung leisten. Zunächst soll der Stand des Wissens zur Entstehung, Diagnostik und Therapie von Erkrankungen und Zuständen mit MR-tomographisch einhergehendem Knochenmarködemmuster geschildert werden (Beitrag Quack). Wir wollen ferner auch das notwendige Bewusstsein für diese Bildmorphologien schaffen und Unfallchirurgen und Radiologen mit einer gemeinsamen, einheitlichen Sprache darüber reden lassen. Das soll am Beispiel der Artikel zu Gelenkverletzungen (Beitrag Rangger), zu den okkulten Frakturen in der Fuß- und Sprunggelenkregion (Beitrag Regauer), zu den Wirbelkörperfrakturen und in einem Fallbericht (Beiträge Geith) verdeutlicht werden.

Wir danken unseren Autoren für ihre umfassende und prägnante Darstellung der einzelnen Themenbereiche, wünschen unseren Lesern eine spannende Beschäftigung mit dem „Knochenmarködem“ und hoffen, damit einen Beitrag zur Sensibilisierung für dieses Thema zu leisten und weitere Diskussionen anzustoßen.

Ihre

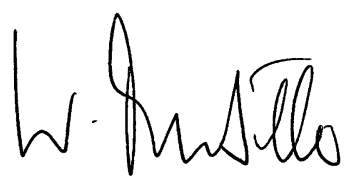

W. Mutschler

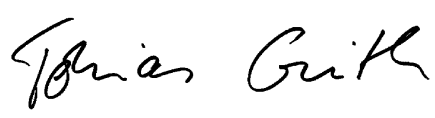

T. Geith

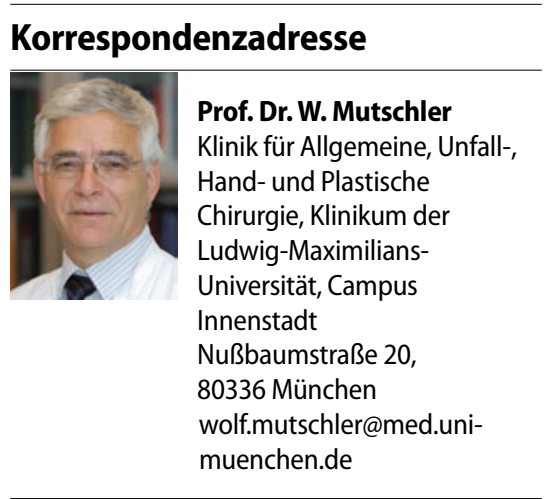

Einhaltung ethischer Richtlinien

Interessenkonflikt. W. Mutschler und T. Geith geben an, dass kein Interessenkonflikt besteht. 